\title{
Dynamically induced scalar quark confinement: A link between chiral symmetry breaking and confinement
}

\author{
Reinhard Alkofer* \\ Institut für Physik, Karl-Franzens-Universität, Universitätsplatz 5, A-8010 Graz, Austria \\ E-mail: reinhard.alkofer@uni-graz.at
}

\section{Christian S. Fischer}

Institut für Physik, TU Darmstadt, Schlossgartenstr. 9, D-64289 Darmstadt, Germany

\section{Felipe J. Llanes-Estrada}

Universidad Complutense de Madrid, Depto. Física Teórica I. 28040 Madrid, Spain

\section{Kai Schwenzer}

Institut für Physik, Karl-Franzens-Universität, Universitätsplatz 5, A-8010 Graz, Austria

\begin{abstract}
Employing functional approaches the infrared behaviour of Landau gauge QCD vertex functions is investigated. Results for the ghost-gluon, three-gluon and quark-gluon vertex functions are presented. As can be analytically shown a linear rising potential between heavy quarks is generated by infrared singularities in the dressed quark-gluon vertex. The selfconsistent mechanism that generates these singularities implies the existence of scalar Dirac amplitudes of the full vertex and the quark propagator. These amplitudes can only be present when chiral symmetry is broken, either explicitly or dynamically. The corresponding relations thus constitute a novel mechanism that directly links chiral symmetry breaking with confinement.
\end{abstract}

The XXV International Symposium on Lattice Field Theory

July 30-4 August 2007

Regensburg, Germany

\footnotetext{
${ }^{*}$ Speaker.
} 


\section{Introduction}

Quark confinement and dynamical chiral symmetry breaking are the two most prominent phenomena of infrared QCD. Recent Monte-Carlo lattice calculations made clear that there is, at least for quarks in the fundamental representation, a close and yet not fully understood relation between these two properties of QCD. E.g. the spectral properties of the Dirac operator reflect both, confinement and chiral symmetry breaking [1]. It is the central aim of this talk to shed light onto this issue from the point of view of QCD Green functions in the Landau gauge.

In this and related functional approaches dynamical chiral symmetry breaking finds a direct explanation [2], the main challenge for such non-perturbative methods is posed by the properties of the linearly rising static quark-antiquark potential. There have been many quite different attempts to relate the properties of this potential to properties of QCD, and thus explain quark confinement. In ref. [3] some of these pictures have been briefly reviewed. These explanations for confinement are seemingly different but there are surprising relations between them which are not yet understood. Given the current status one has to note that these theories are definitely not mutually exclusive but simply reveal only different aspects of the confinement phenomenon. And into one special facet, the above mentioned relation to broken chiral symmetry, there is novel insight from the Landau gauge Greens functions approach.

\section{Infrared Yang-Mills theory in the Landau gauge}

\subsection{Infrared Exponents of Gluons and Ghosts}
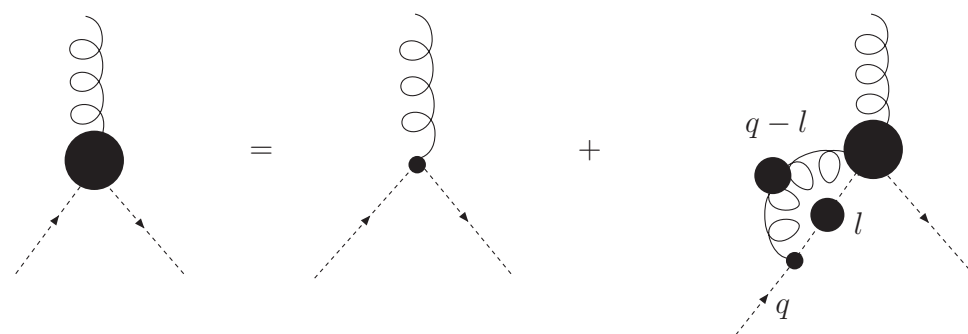

Figure 1: The Dyson-Schwinger equation for the ghost-gluon vertex.

Let us start by looking at the Dyson-Schwinger equation for the ghost-gluon vertex function as depicted in fig. 1. In the Landau gauge the gluon propagator is transverse, and therefore one can employ the relation

$$
l_{\mu} D_{\mu v}(l-q)=q_{\mu} D_{\mu v}(l-q),
$$

to conclude that the ghost-gluon vertex stays finite when the outgoing ghost momentum vanishes, i.e. when $q_{\mu} \rightarrow 0$ [4]. This argument is valid to all orders in perturbation theory, a truely nonperturbative justification of the infrared finiteness of this vertex has been given in refs. $[5,6,7]$.

Using this property of the ghost-gluon vertex the Dyson-Schwinger equation for the ghost propagator, see fig. 2, can be analysed. The only unknowns in the deep infrared are the gluon and the ghost propagators:

$$
D_{\mu v}(k)=\frac{Z\left(k^{2}\right)}{k^{2}}\left(\delta_{\mu v}-\frac{k_{\mu} k_{v}}{k^{2}}\right), \quad D_{G}(k)=-\frac{G\left(k^{2}\right)}{k^{2}} .
$$




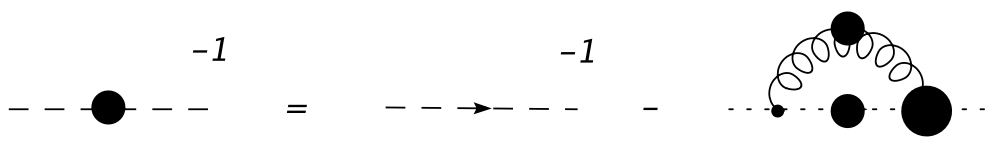

Figure 2: The Dyson-Schwinger equation for the ghost propagator.

In Landau gauge these (Euclidean) propagators are parametrized by two invariant functions, $Z\left(k^{2}\right)$ and $G\left(k^{2}\right)$, respectively. As solutions of renormalized equations, these functions depend also on the renormalization scale $\mu$. Furthermore, assuming that QCD Green functions can be expanded in asymptotic series, the integral in the ghost Dyson-Schwinger equation can be split up in three pieces: an infrared integral, an ultraviolet integral, and an expression for the ghost wave function renormalization. Hereby it is the resulting equation for the latter quantity which allows one to extract definite information [8] without using any truncation or ansatz.

One obtains that the infrared behaviour of the gluon and ghost propagators is given by power laws, and that the exponents are uniquely related such that the gluon exponent is -2 times the ghost exponent [9]. As we will see later on this implies an infrared fixed point for the corresponding running coupling. The signs of the exponents are such that the gluon propagator is infrared suppressed as compared to the one for a free particle, the ghost propagator is infrared enhanced.

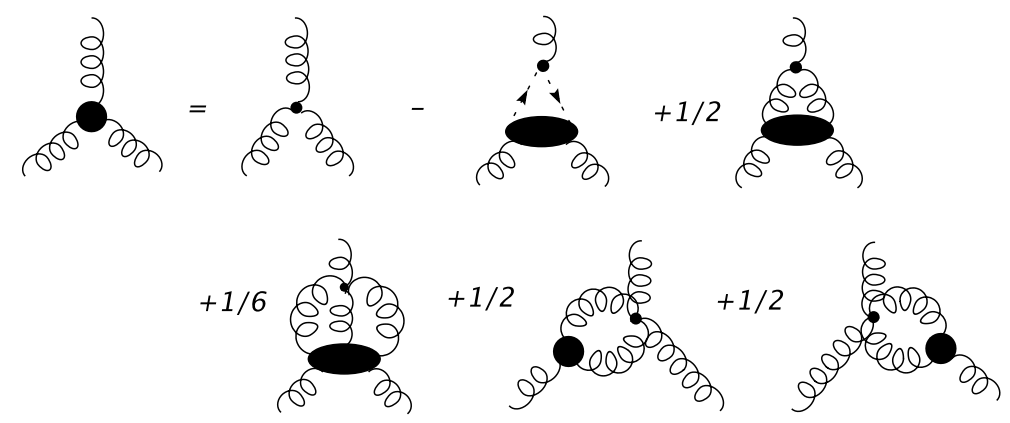

Figure 3: The Dyson-Schwinger equation for the 3-gluon vertex.

Given the infrared power laws, that the Yang-Mills propagators obey, one can infer the infrared behaviour of higher $n$-point functions. To this end the $n$-point Dyson-Schwinger equations have been studied in a skeleton expansion, i.e. a loop expansion using dressed propagators and vertices. Furthermore, an asymptotic expansion has been applied to all primitively divergent Green functions [10]. As an example consider the Dyson-Schwinger equation for the 3-gluon vertex which is diagrammatically represented in fig. 3. Its skeleton expansion, see fig. 4 , can be constructed via the

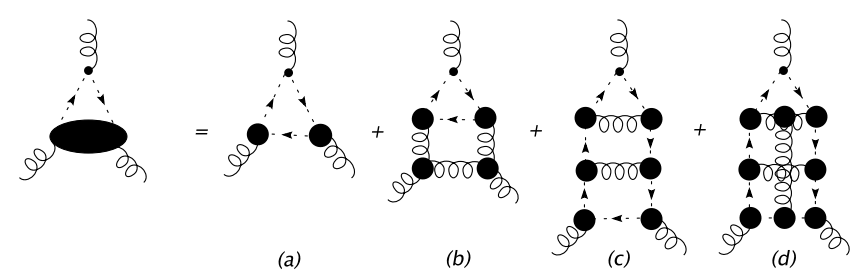

Figure 4: An example for the skeleton expansion of the 3-gluon vertex.

insertions given in fig. 5. These insertions have vanishing infrared anomalous dimensions which implies that the resulting higher order terms feature the same infrared scaling. Based on this the 


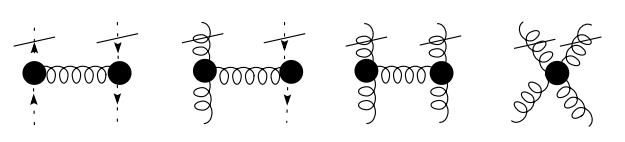

Figure 5: Insertions to reconstruct higher orders in the skeleton expansion.

following general infrared behaviour for one-particle irreducible Green functions with $2 n$ external ghost legs and $m$ external gluon legs can be derived[10, 11]:

$$
\Gamma^{n, m}\left(p^{2}\right) \sim\left(p^{2}\right)^{(n-m) \kappa+(1-n)(d / 2-2)}
$$

where $\kappa$ is one yet undetermined parameter, and $d$ is the space-time dimension. Exploiting DysonSchwinger equations and Exact Renormalization Group Equations one can show that this infrared solution is unique [12].

\subsection{Infrared fixed point of the Yang-Mills running coupling}

The infrared behaviour (2.3) especially includes

$$
G\left(p^{2}\right) \sim\left(p^{2}\right)^{-\kappa}, \quad Z\left(p^{2}\right) \sim\left(p^{2}\right)^{2 \kappa} \quad \Gamma^{3 g}\left(p^{2}\right) \sim\left(p^{2}\right)^{-3 \kappa}, \quad \Gamma^{4 g}\left(p^{2}\right) \sim\left(p^{2}\right)^{-4 \kappa}
$$

and therefore the running couplings related to these vertex functions possess an infrared fixed point:

$$
\begin{aligned}
& \alpha^{g h-g l}\left(p^{2}\right)=\alpha_{\mu} G^{2}\left(p^{2}\right) Z\left(p^{2}\right) \sim \frac{\text { const }_{g h-g l}}{N_{c}}, \quad \alpha^{3 g}\left(p^{2}\right)=\alpha_{\mu}\left[\Gamma^{3 g}\left(p^{2}\right)\right]^{2} Z^{3}\left(p^{2}\right) \sim \frac{\text { const }_{3 g}}{N_{c}}, \\
& \alpha^{4 g}\left(p^{2}\right)=\alpha_{\mu} \Gamma^{4 g}\left(p^{2}\right) Z^{2}\left(p^{2}\right) \sim \frac{\text { const }_{4 g}}{N_{c}} .
\end{aligned}
$$

The infrared value of the coupling related to the ghost-gluon vertex can be computed[5, 13]:

$$
\alpha^{g h-g l}(0)=\frac{4 \pi}{6 N_{c}} \frac{\Gamma(3-2 \kappa) \Gamma(3+\kappa) \Gamma(1+\kappa)}{\Gamma^{2}(2-\kappa) \Gamma(2 \kappa)}
$$

This yields $\alpha^{g h-g l}(0)=2.972$ for $N_{c}=3$ and $\kappa=(93-\sqrt{1201}) / 98 \simeq 0.595353$, which is the value obtained with a bare ghost-gluon vertex.

\subsection{Positivity violation for the gluon propagator}

Positivity violation of the propagator of transverse gluons has been for a long time a conjecture which has been supported recently, see e.g. $[14,15]$ and references therein. The basic feature is hereby the infrared suppression of transverse gluons caused by the infrared enhancement of ghosts. As this behaviour clearly signals the confinement of tranverse gluons [16] it is certainly worth to have a closer look at the underlying analytic structure of the gluon propagator.

Note that the infrared exponent $\kappa$ is an irrational number. Given the infrared power laws this implies already that the gluon propagator possesses a cut on the negative real $p^{2}$ axis. It is possible to fit the solution for the gluon propagator quite accurately without introducing further singularities in the complex $p^{2}$ plane [14]:

$$
Z_{\mathrm{fit}}\left(p^{2}\right)=w\left(\frac{p^{2}}{\Lambda_{\mathrm{QCD}}^{2}+p^{2}}\right)^{2 \kappa}\left(\alpha_{\mathrm{fit}}\left(p^{2}\right)\right)^{-\gamma}
$$


$w$ is a normalization parameter, and $\gamma=\left(-13 N_{c}+4 N_{f}\right) /\left(22 N_{c}-4 N_{f}\right)$ is the one-loop value for the anomalous dimension of the gluon propagator. The running coupling is expressed as [17]:

$$
\alpha_{\mathrm{fit}}\left(p^{2}\right)=\frac{\alpha_{S}(0)}{1+p^{2} / \Lambda_{\mathrm{QCD}}^{2}}+\frac{4 \pi}{\beta_{0}} \frac{p^{2}}{\Lambda_{\mathrm{QCD}}^{2}+p^{2}}\left(\frac{1}{\ln \left(p^{2} / \Lambda_{\mathrm{QCD}}^{2}\right)}-\frac{1}{p^{2} / \Lambda_{\mathrm{QCD}}^{2}-1}\right)
$$

with $\beta_{0}=\left(11 N_{c}-2 N_{f}\right) / 3$. It is important to note that the gluon propagator (2.7) possesses a form such that Wick rotation is possible!

\section{Dynamically induced scalar quark confinement}

The above presented results provide an explanation how gluon confinement works in a covariant gauge, but due to the infrared suppression of the gluon propagator quark confinement seems even more mysterious than ever. To proceed as in the above described studies the Dyson-Schwinger equation for the quark propagator is analyzed with the result that the structure of the quark propagator depends crucially on the quark-gluon vertex $[2,17,18,19,20]$. Therefore a detailed study of this three-point function, and especially its infrared behaviour, is mandatory. Its Dyson-Schwinger equation is diagrammatically depicted in fig. 6, its skeleton expansion in fig. 7. But there is a

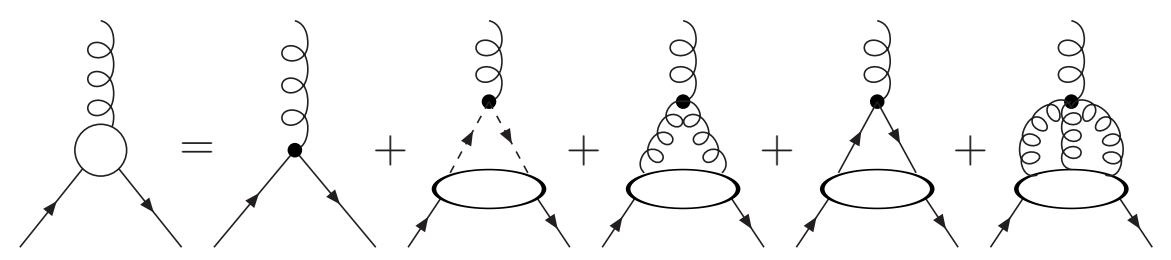

Figure 6: The Dyson-Schwinger equation for the quark-gluon vertex.

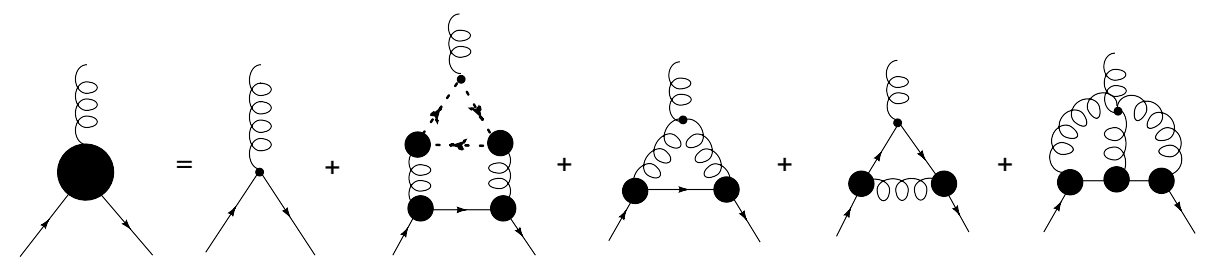

Figure 7: Some leading terms in the skeleton expansion for the quark-gluon vertex.

drastic difference of the quarks as compared to Yang-Mills fields: They possess a current mass. Even if this were not the case one expects dynamical chiral symmetry breaking and thus dynamical mass generation to occur.

To generalize the infrared analysis of the Yang-Mills theory to full QCD [21] one concentrates first on the quark sector of quenched QCD and chooses the masses of the valence quarks to be large, i.e. $m>\Lambda_{\mathrm{QCD}}$. The remaining scales below $\Lambda_{\mathrm{QCD}}$ are those of the external momenta of the propagators and vertex functions. Then the Dyson-Schwinger equations can be used to determine the selfconsistent solutions in terms of powers of the small external momentum scale $p^{2} \ll \Lambda_{\mathrm{QCD}}^{2}$. The equations which have to be considered in addition to the ones of Yang-Mills theory are the one for the quark propagator and the quark-gluon vertex. 
The full quark-gluon vertex $\Gamma_{\mu}$ can consist of up to twelve linearly independent Dirac tensors. Some of those would vanish if chiral symmetry would be realized in the Wigner-Weyl mode: These tensor structures can be non-vanishing either if chiral symmetry is explicitely broken by current masses and/or chiral symmetry is realized in the Nambu-Goldstone mode (i.e. spontaneously broken). From a solution of the Dyson-Schwinger equations we infer that these "Dirac-scalar" structures are, in the chiral limit, generated non-perturbatively together with the dynamical quark mass function in a self-consistent fashion: Dynamical chiral symmetry breaking reflects itself not only in the propagator but also in the quark-gluon vertex function.

From such an infrared analysis one obtains an infrared divergent solution for the quark-gluon vertex such that Dirac vector and "scalar" components of this vertex are infrared divergent with exponent $-\kappa-\frac{1}{2}$ [21]. A numerical solution of a truncated set of Dyson-Schwinger equations confirms this infrared behavior. The driving pieces of this solution are the scalar Dirac amplitudes of the quark-gluon vertex and the scalar part of the quark propagator. Both pieces are only present when chiral symmetry is broken, either explicitely or dynamically.

For the coupling related to the quark-gluon vertex we obtain

$$
\alpha^{q g}\left(p^{2}\right)=\alpha_{\mu}\left[\Gamma^{q g}\left(p^{2}\right)\right]^{2}\left[Z_{f}\left(p^{2}\right)\right]^{2} Z\left(p^{2}\right) \sim \frac{\text { const }_{q g}}{N_{c}} \frac{1}{p^{2}}
$$

using that

$$
\Gamma^{q g}\left(p^{2}\right) \sim\left(p^{2}\right)^{-1 / 2-\kappa}, Z_{f}\left(p^{2}\right) \sim \text { const }, Z\left(p^{2}\right) \sim\left(p^{2}\right)^{2 \kappa} .
$$

Note that the coupling (3.1) is singular in the infrared contrary to the couplings from the Yang-Mills vertices.

In a next step the anomalous infrared exponent of the four-quark function is determined. Note that the static quark potential can be obtained from this four-quark one-particle irreducible Greens function, which, including the canonical dimensions, behaves like $\left(p^{2}\right)^{-2}$ for $p^{2} \rightarrow 0$. Therefore employing the well-known relation for a function $F \propto\left(p^{2}\right)^{-2}$ one obtains

$$
V(\mathbf{r})=\int \frac{d^{3} p}{(2 \pi)^{3}} F\left(p^{0}=0, \mathbf{p}\right) e^{i \mathbf{p r}} \sim|\mathbf{r}|
$$

for the static quark-antiquark potential $V(\mathbf{r})$. We conclude at this point that, given the infrared divergence of the quark-gluon vertex as found in the solution of the coupled system of DysonSchwinger equations, the vertex overcompensates the infared suppression of the gluon propagator, and one therefore obtains a linear rising potential. In addition, this potential is dynamically induced and has a strong scalar component.

To elucidate the here found relation between chiral symmetry breaking and quark confinement we keep chiral symmetry artificially in the Wigner-Weyl mode, i.e. in the chiral limit we force the quark mass term as well as the "scalar" terms in the quark-gluon vertex to be zero. We then find that the resulting running coupling from the quark-gluon vertex is no longer diverging but goes to a fixed point in the infrared similar to the couplings from the Yang-Mills vertices. Correspondingly, one obtains a $1 / r$ behaviour of the static quark potential. The "forced" restoration of chiral symmetry is therefore directly linked with the disappearance of quark confinement. The infared properties of the quark-gluon vertex in the "unforced" solution thus constitute a novel mechanism that directly links chiral symmetry breaking with confinement. 


\section{Summary}

In this talk we have reported on results of functional approaches to infrared QCD in the Landau gauge. We have elucidated the mechanism for gluon confinement: Positivity of transverse gluons is violated. Furthermore, in the Yang-Mills sector the strong running coupling is infrared finite whereas the running coupling from the quark-gluon vertex is infrared divergent. Chiral symmetry is dynamically broken, and this takes place in the quark propagator and the quark-gluon vertex. We have provided clear evidence that static quark confinement in the Landau gauge is due to the infrared divergence of the quark-gluon vertex. In the infrared this vertex has strong scalar components which induce a relation between confinement and broken chiral symmetry.

\section{Acknowledgements}

RA thanks the organisers of Lattice 2007 for all their efforts which made this extraordinary conference possible. We are grateful to A. Cucchieri, A. Maas, T. Mendez, J. Pawlowski, J. Skullerud, and L. v. Smekal for interesting discussions. This work was supported by the DFG under grant no. Al 279/5-2, by the Helmholtz-University Young Investigator Grant VH-NG-332, by the FWF under contract M979-N16, and by MEC travel grant PR2007-0110, Spain.

\section{References}

[1] C. Gattringer, Phys. Rev. Lett. 97, 032003 (2006) [arXiv:hep-lat/0605018];

E. Bilgici, C. Hagen, F. Bruckmann and C. Gattringer, arXiv:0710.0294 [hep-lat].

[2] C. D. Roberts and A. G. Williams, Prog. Part. Nucl. Phys. 33 (1994) 477 [arXiv:hep-ph/9403224].

[3] R. Alkofer and J. Greensite, J. Phys. G34 (2007) S3 [arXiv:hep-ph/0610365].

[4] J. C. Taylor, Nucl. Phys. B33 (1971) 436.

[5] C. Lerche and L. von Smekal, Phys. Rev. D 65 (2002) 125006 [arXiv:hep-ph/0202194].

[6] A. Cucchieri, T. Mendes, and A. Mihara JHEP 12 (2004) 012 [arXiv:hep-lat/0408034].

[7] W. Schleifenbaum et al., Phys. Rev. D72 (2005) 014017 [arXiv:hep-ph/0411052].

[8] P. Watson and R. Alkofer, Phys. Rev. Lett. 86 (2001) 5239 [arXiv:hep-ph/0102332].

[9] L. von Smekal, R. Alkofer and A. Hauck, Phys. Rev. Lett. 79 (1997) 3591 [arXiv:hep-ph/9705242]; L. von Smekal, A. Hauck and R. Alkofer, Annals Phys. 267 (1998) 1 [arXiv:hep-ph/9707327].

[10] R. Alkofer, C. S. Fischer and F. J. Llanes-Estrada, Phys. Lett. B 611 (2005) 279 [arXiv:hep-th/0412330]; R. Alkofer et al., arXiv:nucl-th/0601032.

[11] M. Huber, R. Alkofer, C. S. Fischer and K. Schwenzer, arXiv:0705.3809 [hep-ph].

[12] C. S. Fischer and J. M. Pawlowski, Phys. Rev. D 75 (2007) 025012 [arXiv:hep-th/0609009].

[13] C. S. Fischer and R. Alkofer, Phys. Lett. B536 (2002) 177 [arXiv:hep-ph/0202202]; C. S. Fischer, R. Alkofer and H. Reinhardt, Phys. Rev. D65 2002094008 [arXiv:hep-ph/0202195]; R. Alkofer, C. S. Fischer and L. von Smekal, Acta Phys. Slov. 52(2002) 191 [arXiv:hep-ph/0205125].

[14] R. Alkofer et al., Phys. Rev. D 70 (2004) 014014 [arXiv:hep-ph/0309077]; Nucl. Phys. Proc. Suppl. 141 (2005) 122.

[15] P. O. Bowman et al., arXiv:hep-lat/0703022.

[16] L. von Smekal and R. Alkofer, arXiv:hep-ph/0009219.

[17] C. S. Fischer and R. Alkofer, Phys. Rev. D 67 (2003) 094020 [arXiv:hep-ph/0301094].

[18] R. Alkofer and L. von Smekal, Phys. Rept. 353 (2001) 281 [arXiv:hep-ph/0007355].

[19] C. S. Fischer, J. Phys. G: Nucl. Part. Phys. 32 (2006) R253 [arXiv:hep-ph/0605173].

[20] R. Alkofer, Brazilian J. Phys. 37N1B (2007) 144 [arXiv:hep-ph/0611090].

[21] R. Alkofer, C. S. Fischer and F. J. Llanes-Estrada, arXiv:hep-ph/0607293; R. Alkofer, C. S. Fischer, F. J. Llanes-Estrada, and K. Schwenzer, in preparation. 